Western University

Scholarship@Western

Summer 2019

\title{
Gut yeasts do not improve desiccation survival in Drosophila melanogaster
}

Joanne M. Tang

Western University, jtang283@uwo.ca

Yanira Jiménez Padilla

Western University, yjimenez@uwo.ca

Marc-André Lachance

Western University, lachance@uwo.ca

Brent J. Sinclair

Western University, bsincla7@uwo.ca

Follow this and additional works at: https://ir.lib.uwo.ca/biologypub

Part of the Biology Commons

\section{Citation of this paper:}

Tang, Joanne M.; Jiménez Padilla, Yanira; Lachance, Marc-André; and Sinclair, Brent J., "Gut yeasts do not improve desiccation survival in Drosophila melanogaster" (2019). Biology Publications. 105.

https://ir.lib.uwo.ca/biologypub/105 
1 Gut yeasts do not improve desiccation survival in Drosophila melanogaster

3 Joanne M. Tang ${ }^{1}$, Yanira Jiménez-Padilla, Marc-André Lachance, and Brent J. Sinclair*

4

5

6

7 Department of Biology, University of Western Ontario, London, ON, Canada N6G 1L3

8

$9{ }^{1}$ Present Address: Department of Medical Biophysics, University of Western Ontario, London,

10 ON, Canada.

$11 *$ *Corresponding Author: email bsincla7@uwo.ca, tel: +1-519-661-2111 x83138

12 


\section{Research Highlights:}

17

18

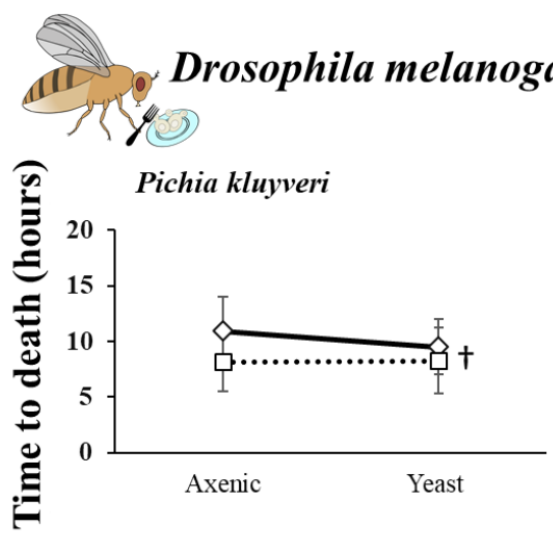

Graphical Abstract:
- Gut yeasts do not improve adult Drosophila melanogaster desiccation survival

- Rearing flies with non-coevolved yeasts reduces adult desiccation survival

- Saccharomyces cerevisiae may not be a beneficial contributor to the gut microbiota

19
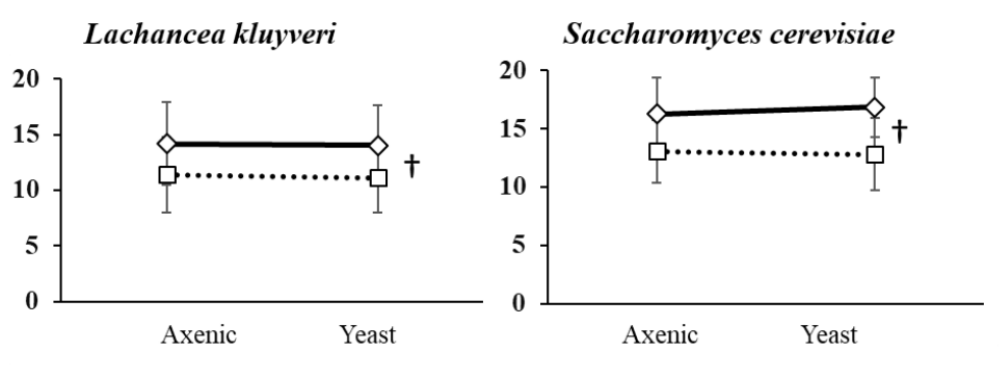
21 A healthy gut microbiota generally improves the performance of its insect host. Although the

22 effects can be specific to the species composition of the microbial community, the role of gut

23 microbiota in determining water balance has not been well-explored. We used axenic and

24 gnotobiotic (reared with a known microbiota) Drosophila melanogaster to test three hypotheses

25 about the effects of gut yeasts on the water balance of adult flies: 1) that gut yeasts would

26 improve desiccation survival in adult flies; 2) that larval yeasts would improve adult desiccation

27 survival; 3) that the effects would be species-specific, such that yeasts closely associated with $D$. melanogaster in nature are more likely to be beneficial than those rarely found in association

29 with D. melanogaster. We used Saccharomyces cerevisiae (often used in Drosophila cultures, but rarely associated with $D$. melanogaster in nature), Lachancea kluyveri (associated with some

31 species of Drosophila, but not D. melanogaster), and Pichia kluyveri (associated with D.

32 melanogaster in nature). Adult inoculation with yeasts had no effect on survival of desiccating conditions. Inoculation with P. kluyveri as larvae, did not change desiccation survival in adults;

34 however, rearing with L. kluyveri or S. cerevisiae reduced adult desiccation survival. We conclude that adult inoculation with gut yeasts has no impact on desiccation survival, but that rearing with yeasts can have either no or detrimental effect. The effects appear to be species-

37 specific: P. kluyveri did not have a negative impact on desiccation tolerance, suggesting some

38 level of co-adaptation with D. melanogaster. We note that S. cerevisiae may not be an

39 appropriate species for studying the effects of gut yeasts on D. melanogaster.

41 Key Words: Desiccation; gut microbiota; phenotypic plasticity; Pichia kluyveri;

42 Lachancea kluyveri; Saccharomyces cerevisiae 


\section{Abbreviations:}

$44 \quad$ FM: Fresh mass

45 MAD: Mass at death

46 DM: Dry mass

47 IWC: Initial water content

48 WCD: Water content at death

49 WLR: Water loss rate

50 
52 Animals have an abundant and diverse microbiota that can have profound impacts on their 53 physiology, reproduction, and behaviour (McFall-Ngai et al., 2013). Although most epithelia 54 have a distinct microbiota, and some microbial symbionts are intracellular, a majority of 55 microbes colonise the gut (Douglas, 2018b). Insects are tractable models for studying the effects 56 of this gut microbiota on performance (Douglas, 2015), not least because of the ease with which 57 aseptic rearing and inoculation can yield axenic (no microbiota) and gnotobiotic (with a known 58 microbiota) individuals. The gut microbiota has profound effects on digestion (Peterson and 59 Scharf, 2016), toxin breakdown (Welte et al., 2016), immunity (Dillon and Charnley, 1986), 60 nutrition (Scully et al., 2014) and behaviour (Wada-Katsumata et al., 2015), and can modify 61 development time and cold tolerance (Jiménez Padilla, 2016). These effects can be context62 dependent. For example, microbe inoculation during development increases Drosophila lifespan, 63 but inoculation as an adult reduces lifespan (Brummel et al., 2004). Moreover, some gut 64 microbes have a greater impact than others (Douglas, 2018b; Newell et al., 2014). In general, 65 however, those individuals with a gut microbiota tend to outperform axenic individuals that lack 66 gut microbes (Douglas, 2015; but see Henry and Colinet, 2018; Judd et al., 2018).

68 The gut microbiota of insects includes bacteria, archaea, viruses, and yeasts (Douglas, 2015).

69 While a majority of studies have focused on bacteria (Engel and Moran, 2013), there is a rich

70 yeast community associated with insects, including with Drosophila spp. (e.g. Anagnostou et al.,

71 2010; Lachance et al., 1995; Starmer and Fogleman, 1986). More than 56 species of yeasts have

72 been identified from the guts of wild-caught Drosophila spp. (Lachance et al., 1995); however

73 Saccharomyces cerevisiae, the standard yeast used in Drosophila spp. rearing (Ashburner et al., 
74 2005; Markow and O'Grady, 2005), is not one of the species regularly identified in the guts of

75 wild-caught flies (Chandler et al., 2012) and may not be a D. melanogaster symbiont (Hoang et

76 al., 2015). Jiménez Padilla (2016) showed that yeasts primarily colonise the guts of female $D$.

77 melanogaster, that $S$. cerevisiae persisted in the gut for less time than Lachancea kluyveri [a

78 yeast collected from D. robusta, D. pinicola, and D. algonquin in the wild (Lachance et al.,

79 1995; Phaff et al., 1956)], and that L. kluyveri had a larger effect on D. melanogaster chill coma

80 recovery time than $S$. cerevisiae. Thus, just as with gut bacteria (Newell et al., 2014), the

81 phenotypic effects of gut yeasts on Drosophila may be species-specific, and may not stem only

82 from the additional nutrients acquired from digesting the yeast, as has previously been suggested

83 (Anagnostou et al., 2010; Shin et al., 2011).

85 The ability to maintain water balance under desiccating conditions has been key to the success of terrestrial insects (Harrison et al., 2012). Insects that survive desiccating conditions for longer

87 generally use some combination of increased initial water content, reduced water loss rate, and tolerating the loss of more water (Gibbs, 2002; Gibbs et al., 1997; Gibbs and Matzkin, 2001). Drosophila spp. that best survive desiccation, or populations of D. melanogaster selected to 90 survive for longer, use the first two of these strategies. Water loss rate across the cuticle can be modified by changing the cuticular hydrocarbons to yield plasticity even within a population

92 (Bazinet et al., 2010; Hoffmann, 1990; Stinziano et al., 2015). Dung beetles reared under desiccating conditions have better survival, faster growth rates, and larger final body size when

94 they have a full, rather than reduced, microbiota (Schwab et al., 2016), although the mechanisms 95 underlying this difference were not determined. Thus, the role - if any - of the gut microbiota 96 (and especially yeasts) on insect water balance remains largely unexplored. 
98 Here we examined the effects of gut yeasts on desiccation survival of adult D. melanogaster to 99 better understand the role of gut yeasts in insect water balance. First, we hypothesised that gut 100 yeasts improve desiccation survival. We compared the survival time of axenic and gnotobiotic 101 flies under desiccating conditions, and measured water content, desiccation tolerance, and water 102 loss rates to examine the mechanisms underlying any differences. Second, we hypothesised that 103 development with gut yeasts improves desiccation survival in adults. We reared axenic and 104 gnotobiotic larvae, and then compared their survival as adults under desiccating conditions.

105 Finally, we hypothesised that the effects of the gut community on performance under desiccating 106 conditions are species-specific. We compared gnotobiotic flies inoculated with Pichia kluyveri 107 [which is regularly found in D. melanogaster guts in nature (Lachance et al., 1995)], Lachancea 108 kluyveri [which has a strong phenotypic effect on D. melanogaster development time and cold 109 tolerance (Jiménez Padilla, 2016), but is not normally found in D. melanogaster in nature], and 110 Saccharomyces cerevisiae, which is seldom found in D. melanogaster in nature, but is an 111 ubiquitous additive in laboratory Drosophila cultures (Ashburner et al., 2005; Markow and 112 O'Grady, 2005). 


\section{Methods}

115

116

117

118

119

120

121

122

123

124

125

126

127

128

129

130

131

132

133

134

135

136

26

\subsection{Study Organisms}

We used an outbred, wild-type Drosophila melanogaster population collected in London,

Ontario, Canada $\left(43^{\circ} 00^{\prime} \mathrm{N}, 81^{\circ} 15^{\prime} \mathrm{W}\right)$ in 2007 (Marshall and Sinclair, 2010). We reared the flies in $35 \mathrm{~mL}$ vials containing $20 \mathrm{~mL}$ of Tucson fly food [1 L d $\mathrm{d}_{2} \mathrm{O}, 45 \mathrm{~g}$ sugar, $30 \mathrm{~g}$ cornmeal, $18 \mathrm{~g}$ dry active S. cerevisiae (Fleischmann's Yeast, Farinex, QC, Canada), 12 g agar; adapted from Markow and O'Grady (2005)] under standard long-day conditions $\left(21.5 \pm 1{ }^{\circ} \mathrm{C}, 60 \pm 5 \% \mathrm{RH}, 13\right.$ h:11 h L:D) at standardized densities (50 eggs/vial). We transferred three- to five-day old adult flies into small oviposition cages $(\varnothing=3.5 \mathrm{~cm}, \mathrm{~h}=5.8 \mathrm{~cm})$ capped with a Petri dish of apple juice-

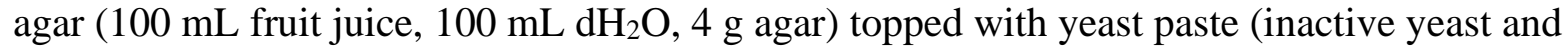
distilled water) for three days. For egg collection, we replaced the yeast-topped apple-agar plates with apple-agar plates on which the flies laid eggs for $12 \mathrm{~h}$.

To generate axenic flies, we transferred fifty eggs onto autoclaved sterile $20 \mu \mathrm{m}$ pore nylon

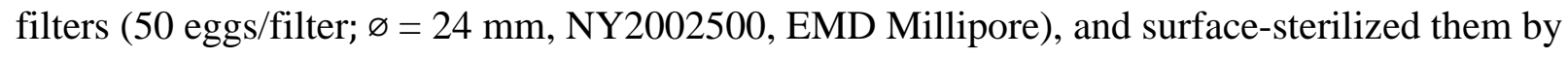
submerging them in $70 \%$ ethanol for 5 min and rinsing them with autoclaved phosphate buffered saline (PBS) (Sigma-Aldrich, P4417, prepared as per manufacturer's instructions). Each filter was then inverted onto a sterile yeast-sucrose agar plate (1.5 g agar, $1.5 \mathrm{~g}$ active yeast, $4.3 \mathrm{~g}$

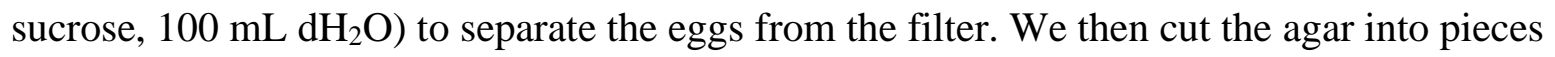
containing the eggs (still 50/vial) and transferred the pieces to autoclaved $35 \mathrm{~mL}$ vials each containing $10 \mathrm{~mL}$ of Tucson fly food (the active yeasts in the recipe are killed when the food is autoclaved). Vials were incubated under standard conditions until adult emergence. We confirmed that the adult flies in each vial were axenic prior to use in experiments. We narcotized 
137 flies with $\mathrm{CO}_{2}$, and collected two flies of each sex and a small sample of food surface from each

138 vial. We homogenized these samples in $100 \mu \mathrm{L}$ sterile PBS, plated them on Yeast-Malt agar

139 (YM agar) (1\% glucose, $0.5 \%$ peptone, $0.3 \%$ malt extract, $0.3 \%$ yeast extract, $2 \%$ agar), and

140 incubated them at $25^{\circ} \mathrm{C}$ for $48 \mathrm{~h}$. All flies reared in vials showing any microbial growth after 48

$141 \mathrm{~h}$ were discarded from the experiment. In practice, we had 85-90\% success in maintaining sterile

142 conditions, even though we had several instances of transferring flies. The remaining axenic flies

143 were transferred into new $35 \mathrm{~mL}$ vials of sterile Tucson fly food for experiments.

145 To make adult-inoculated gnotobiotic flies, we took axenic adults, and allowed them to feed on

146 live yeasts for $48 \mathrm{~h}$; controls were provided with PBS or heat-killed yeasts at the same

147 concentration. Yeasts were Pichia kluyveri (strain UWOPS 91-603.2), Lachancea kluyveri

148 (strain NRRL.Y-12651), and Saccharomyces cerevisiae (strain UWOPS 92-222.2) obtained from

149 the yeast collection of the Department of Biology, University of Western Ontario. We pipetted

$15010 \mu \mathrm{L}$ of a suspension of $1.3 \times 10^{8}$ cells $/ \mathrm{mL}$ in PBS (or $10 \mu \mathrm{L}$ of sterile PBS, for controls) prior to

151 adding axenic flies. Flies were allowed to feed on the yeast for $48 \mathrm{~h}$ at $21.5^{\circ} \mathrm{C}$ prior to use in

152 experiments. To confirm that the gnotobiotic flies were colonised with yeasts, we removed two

153 male and two female flies from each yeast-inoculated vial, surface sterilised them in $70 \%$

154 ethanol, rinsed them twice in sterile PBS, and then homogenized them in $100 \mu \mathrm{L}$ of sterile PBS.

155 We plated the homogenate on YM agar, incubated for $48-72 \mathrm{~h}$ at $25^{\circ} \mathrm{C}$, and checked for yeast

156 growth (vials without yeast growth, or with noticeable bacteria growth, were removed from the

157 experiment). The remaining flies from each vial were used in desiccation experiments. We

158 reared flies under gnotobiotic conditions by adding $10 \mu \mathrm{L}$ of yeast suspension into vials

159 containing $10 \mathrm{~mL}$ of sterile Tucson food before transferring fifty surface-sterilised eggs to each 
160 vial and incubating them under standard rearing conditions. Checks for contamination were

161 conducted as described above, and contaminated vials discarded from the experiments.

163 As a control for the nutritional contribution of yeasts in the gnotobiotic treatment, we reared

164 flies with heat-killed yeasts. We heat-killed yeast suspensions $\left(1.3 \times 10^{8}\right.$ cells $/ \mathrm{mL}$ in PBS $)$ by

165 heating them at $60{ }^{\circ} \mathrm{C}$ for $30 \mathrm{~min}$. We confirmed that the yeasts were dead by plating $10 \mu \mathrm{L}$ of

166 the suspension on YM agar plates before transferring fifty surface-sterilised eggs to vials

167 containing $10 \mathrm{~mL}$ sterile Tucson food inoculated with $10 \mu \mathrm{L}$ of heat-killed yeast suspension and

168 incubating as above. As above, vials showing bacterial or yeast growth were discarded from the

169 experiments.

170

$171 \quad 2.2$ Desiccation survival and water balance parameters

172 We measured survival under desiccating conditions as described by Gefen et al. (2006) and

173 Bazinet et al. (2010). Briefly, we transferred flies individually into empty non-sterile $35 \mathrm{~mL}$ vials

174 without anaesthesia (one fly per vial, $\mathrm{n}>28$ flies per treatment, 28-33 vials/treatment, based on a

175 power analysis from pilot experiments). We assumed that the duration of the desiccation

176 experiment was too short for the flies to form a functioning gut microbiota from an empty vial,

177 and that the mass lost was primarily water. We confined the fly to the bottom half of the vial

178 using a foam stopper and added approximately $2 \mathrm{~g}$ of silica gel before sealing the vial with

179 parafilm. We recorded survival (ability to right themselves) at hourly intervals until all flies were

180 dead. To control for starvation, we separated 28-33 flies from different vials individually into

181 vials containing water-agar medium and observed no mortality over the duration of the

182 experiment, which suggests that handling and starvation did not cause mortality in either the 
183 axenic or gnotobiotic. To control for variation between experimental days, experiments

184 conducted on the same date were denoted with the same cohort number and we included cohort 185 as a term in models.

187 We determined initial water content, water content at death, and water loss rate gravimetrically. 188 Briefly, we weighed each fly (MX5 microbalance, Mettler Toledo, Columbus, OH, USA) before 189 the start of the experiment to obtain fresh mass (FM), after the desiccation experiment to obtain 190 mass at death (MAD), and after drying at $60{ }^{\circ} \mathrm{C}$ for $48 \mathrm{~h}$ to obtain dry mass (DM). Initial water 191 content (IWC) was calculated as the difference between FM and DM; water content at death 192 (WCD) as the difference between MAD and DM, and water loss rate (WLR) as the difference 193 between IWC and WCD divided by time to death (yielding an average rate in $\mathrm{mg} \mathrm{H}_{2} \mathrm{O} / \mathrm{hour}$ ).

195 We compared survival time under desiccating conditions, initial water content, water content at 196 death, and water loss rate between axenic and gnotobiotic flies separately for each yeast and 197 treatment (reared or adult-inoculated) using two-way analyses of covariance (ANCOVA) in R 198 (version 3.2.2), with dry mass, sex, and cohort (the date of the experiment) as covariates. We 199 chose to use this linear model approach to compare survival times (cf. Gibbs et al., 1997) instead 200 of a survival analysis (cf. Jakobs et al., 2015) as it allowed us to include cohort effects in our 201 models. However, we provide survival curves and log-rank analyses, which are consistent with 202 these results, in supplementary material). We included only individuals for which all 203 measurements were available and discarded any for which measurement errors resulted in 204 impossible values (e.g. negative water content). We corrected all p-values using a study-wide 
205 Benjamini-Hochberg FDR correction (Benjamini and Hochberg, 1995) using the p.adjust 206 function in R. 


\section{Results}

208

209

210

211

212

213

214

215

216

217

218

219

220

221

222

223

224

225

226

227

Axenic flies were consistently sterile, and we were able to culture the expected yeasts from adult-inoculated gnotobiotic flies as well as gnotobiotic-reared flies (example culture plates in Figures S1 and S2). Female flies and larger flies survived desiccation significantly longer than males or smaller flies in all groups and treatments.

\subsection{Gut yeasts do not improve desiccation survival in adult-inoculated flies}

Neither female nor male D. melanogaster inoculated as adults with any of the species of yeasts survived desiccation stress significantly longer than their axenic counterparts (Figure 1; Table 1; Figs S3-S5), although a survival analysis (that considers the entire curve) showed that one cohort of P. kluyveri-inoculated female flies had worse survival than their axenic counterparts. There was no sex $\times$ treatment interaction, but there was a significant cohort effect, leading us to retain this term in our model.

Despite this overall lack of effects of yeasts on desiccation survival, gut yeasts did change some water balance parameters. Inoculation with any of the yeasts did not change initial water content (Figure 2A, D, G; Table 1). Water content at death was not significantly affected by any of the three yeast treatments (Figure 2B, E, H; Table 1). Inoculation with any of the yeasts had no significant impact on water loss rates (Figure 2C, F, I; Table 1).

\subsection{Gnotobiotic rearing with yeast does not improve desiccation survival}


228 Female and male adults reared with $P$. kluyveri did not have significantly different survival time

229 under desiccating conditions than their axenic counterparts, but rearing with either Lachancea

230 kluyveri or $S$. cerevisiae significantly decreased survival time under desiccating conditions

231 compared to axenic flies in both females and males (Figure 3; Table 2; Figure S7). Flies reared

232 with yeasts of all three species had significantly higher initial water contents than their axenic

233 counterparts, even after accounting for the effects of body size and sex (Figure 4A, D, G; Table

234 2). However, the gnotobiotic flies reared with L. kluyveri and S. cerevisiae (but not P. kluyveri)

235 had higher water loss rates than their axenic counterparts (Figure 4C, F, I; Table 2). In addition,

236 both males and females of flies gnotobiotically-reared with P. kluyveri and L. kluyveri had higher

237 water contents at death than their axenic counterparts, but there was no effect of yeast rearing on

238 the water content at death of gnotobiotic flies reared with S. cerevisiae (Figure 4B, E, H; Table

$2392)$.

240

241 Rearing with heat-killed yeasts did not change the differences between male and female flies, but

242 rearing with dead $S$. cerevisiae did slightly increase desiccation survival time in females (Figure

243 3; Table 3), although this was not significant in a survival analysis (Figure S8). Rearing with

244 heat-killed yeasts increased water content at death in flies inoculated with both $P$. kluyveri and $S$.

245 cerevisiae, decreased rate of water loss in S. cerevisiae, and slightly increased initial water

246 content in flies reared with dead S. cerevisiae. However, rearing with heat-killed L. kluyveri had

247 no impact on water balance parameters (Figure 5; Table 3). 


\section{Discussion}

250 Investigations of the impact of gut microbiota on insect phenotypes have generally focused on 251 bacteria (Douglas, 2018a), and in these studies, insects with a gut microbiota usually outperform 252 their axenic counterparts (Coon et al., 2014; Wong et al., 2014). Here we investigate the role of 253 gut yeasts on the water balance of Drosophila melanogaster. We find that inoculation with 254 yeasts does not improve desiccation survival of adult flies, and that flies reared with live yeasts 255 have reduced desiccation survival relative to their axenic counterparts. This impact is species256 specific, and it appears that yeast species not usually associated with $D$. melanogaster in nature 257 including S. cerevisiae, which is widely used in Drosophila culture - are more likely to have a 258 deleterious impact.

Desiccation survival by adult $D$. melanogaster is plastic at several timescales, ranging from brief

261 'hardening' responses (Bazinet et al., 2010; Hoffmann, 1990) to longer term responses to 262 selection (Gibbs et al., 1997). Although live yeasts will colonise the guts of adult flies (Jiménez 263 Padilla, 2016), we found no impact of inoculation with live yeasts on survival time under 264 desiccating conditions. Inoculation with live $S$. cerevisiae did increase initial water content, but 265 this was offset by a slightly increased water loss rate, yielding a survival time indistinguishable 266 from axenic flies, or those inoculated with L. kluyveri or P. kluyveri. We do not have immediate 267 hypotheses to explain these effects of S. cerevisiae, or why we saw the effect in flies inoculated 268 with $S$. cerevisiae. Because this variation does not appear to affect desiccation survival, we do 269 not explore it further here. 
271 Larval nutrition can have far-reaching effects on adult performance (e.g. Vijendravarma et al.,

272 2010). Competition for dietary yeasts mediates the effects of larval crowding on a range of traits,

273 including structural body size (Klepsatel et al., 2018), which is an important determinant of

274 desiccation survival in Drosophila (Gibbs and Matzkin, 2001). We found that flies reared with

275 live S. cerevisiae and L. kluyveri survived desiccation for less time than their axenic

276 counterparts, and we showed that the yeasts had to be alive to have this effect. Furthermore, we

277 accounted for body size in our analyses, which suggests that the effects we see are not simply a

278 consequence of larval food resources yielding different-sized flies with corresponding variation

279 in capacity to maintain water balance. Thus, we conclude that the effects of yeasts we observed

280 on adult flies were not mediated by variation in yeast nutritional availability during larval

281 development.

Desiccation survival in Drosophila is not usually associated with changes in desiccation

284 tolerance (i.e. water content at death; Gibbs, 2002; Gibbs et al., 1997; Gibbs et al., 2003; Gibbs

285 and Matzkin, 2001), so it is surprising to see that some rearing conditions modify water content

286 at death in adult flies. One possible explanation for this effect is that the live yeasts in the gut

287 results in a net increase in whole body water content, but that the increased bulk water in the

288 yeast cells is not available to the fly. Alternately, the increased body water content could lead to

289 adult flies that do not regulate water balance as tightly, leading to lower survival when exposed

290 to desiccating conditions. While water loss rates can be plastic (e.g. Bazinet et al., 2010) and

291 could yield this result, we are not aware of corresponding plasticity in the amount of water loss

292 tolerated in Drosophila. Relatively little is known about the cellular mechanisms causing death

293 from dehydration in insects (Bradley, 2009; Gibbs et al., 1997; Hadley, 1994; Harrison et al., 
294 2012), which prevents us from speculating about the mechanistic link between gut yeast and 295 desiccation tolerance in our experiments. However, we do not see equivalent effects of rearing 296 with dead yeasts on water balance parameters or desiccation survival, which leads us to conclude 297 that the impact of gut yeasts on water balance is dependent on the yeasts being alive, and either 298 due to modification of digestive processes in the gut (cf. Wong et al., 2014), or a direct 299 biological interaction between the yeast and its host (cf. Broderick et al., 2014; Schretter et al., 300 2018).

The effects of yeast rearing on both desiccation survival and individual water balance parameters 303 in adult D. melanogaster appear to be species-specific. Pichia kluyveri is found in D.

304 melanogaster guts in nature, and although it does not improve performance in our experiments, it 305 is the only species we tested that was not in some way detrimental to desiccation survival, 306 supporting the hypothesis that there is some degree of yeast-host coevolution, as suggested by 307 Starmer and Fogleman (1986). Our results were largely consistent across cohorts for P. kluyveri 308 and L. kluyveri, with the one exception being in female adults where inoculation with $P$. kluyveri was detrimental to desiccation survival in one cohort, but not the other. However, as our

310 prediction was that yeast would improve desiccation survival, this does not modify our 311 conclusion that gut yeasts do not improve desiccation survival. Rearing with P. kluyveri still 312 increased both initial water content and water content at death in adult flies, which we suggest 313 provides support for our contention that the increased water content we measured is not 314 physiologically available to the fly. This hypothesis could be tested in the future using stable315 isotope labelled water in the yeasts (cf. McCluney and Sabo, 2010). Lachancea kluyveri has not 316 been collected from D. melanogaster in nature (although it is found in other Drosophila species), 
317 and has positive impacts on both development time and chill coma recovery (Jiménez Padilla,

318 2016). Thus, the identification of improved performance in one or two traits (development time

319 and chill coma) does not necessarily imply that the same yeast is beneficial for all traits

320 (desiccation tolerance in this case). This implies that the choice of traits for studying host-

321 microbe interactions is important when extrapolating to fitness or field performance.

323 In nature (and indeed, under normal circumstances in the laboratory), Drosophila larvae grow in 324 a microbe-rich environment including abundant yeasts of many species, alongside bacteria and 325 other microbes (Broderick and Lemaitre, 2012; Chandler et al., 2012; Wong et al., 2011). Our 326 single-yeast gnotobiotic system is clearly unnatural (and we cannot rule out more complex 327 interactions among diverse gut microbiota). It nonetheless allows us to demonstrate that gut 328 yeasts can have an effect, and that the identity of those yeasts appears to be important in 329 determining host performance (cf. Ben Ami et al., 2010). Although we report what appear to be 330 negative (or at best neutral) consequences of yeast-fly coexistence, there appears to be 331 coevolution in the wild (Starmer and Fogleman, 1986), and some of the same yeasts that have 332 negative consequences for water balance in this study have positive impacts on other traits 333 (Jiménez Padilla, 2016). Indeed, flies in the wild seek out yeast preferentially (Becher et al., 334 2012; Dobzhansky et al., 1956). We propose two non-mutually exclusive explanations for this 335 effect. First, the effects of gut microbes can be context-dependent, for example conventional flies 336 develop faster and store less lipid than axenic flies only in high-glucose, low-yeast diets (Wong 337 et al., 2014). Thus, it is possible that yeasts are beneficial - even to water balance - in a field 338 context that is not adequately captured in our laboratory experiments. Second, the water balance consequence of larval development with yeast may be inconsequential compared to the benefits 
340 accrued to development time and thermal performance (Jiménez Padilla, 2016), such that yeast-

341 host interactions are maintained.

Baker's yeast, Saccharomyces cerevisiae, is a standard ingredient in most Drosophila rearing

344 media (Ashburner et al., 2005; Markow and O'Grady, 2005), even though it is seldom collected

345 from Drosophila in nature (Hoang et al., 2015). We found that inoculation with S. cerevisiae had

346 no impact on desiccation survival or water balance parameters. By contrast, larval rearing with $S$.

347 cerevisiae decreased adult desiccation survival, increased initial water content, and slightly

348 decreased water loss rate. These observations have two important implications. First,

349 'conventional' rearing with $S$. cerevisiae may have impacts that are not reflected in the biology

350 of Drosophila in the field that primarily interact with other species of yeast. However, we do

351 note that in our laboratory, where our conventionally-reared flies receive live $S$. cerevisiae, the

352 predominant culturable yeast species from the vials is an as-yet unidentified species (Jiménez-

353 Padilla and Lachance, unpublished observations). Thus, the overall microbiological milieu of

354 human-built environments may be providing some inadvertent correction for our dependence on

355 S. cerevisiae in Drosophila cultures, although we have not explored the yeast community of

356 Drosophila cultures beyond our own laboratory to see if this is a widespread effect. Second,

357 because it has phenotypic effects different to other yeasts, and is not normally associated with $D$.

358 melanogaster in nature, $S$. cerevisiae may be an inappropriate yeast for experiments on gut yeast

359 in insects (at least without direct comparison to other species of yeast more closely associated

360 with the host; see also Hoang et al., 2015). 
362 In conclusion, gut yeasts during development can affect water balance of adult D. melanogaster,

363 but generally this effect is detrimental, appears to require long-term exposure to the yeasts during

364 larval development, and depends on the yeasts being alive. There are among-species differences

365 in the phenotypic effect of gut yeasts on water balance which are broadly consistent with some

366 degree of co-evolution between yeast species and their insect hosts. Finally, we support the

367 assertions of Hoang et al. (2015) that S. cerevisiae may not be the most appropriate species of

368 yeast to use when examining the effects of gut yeasts on Drosophila biology. 


\section{Acknowledgements}

371 We thank Trish Tully for discussion, and Dr. Jim Staples for use of some equipment. Thanks to

372 Adam Smith, Dina Mostafa, Eddy Huang, SongMin Lee, and Sana Homsi who assisted in the

373 laboratory. We are grateful to two anonymous reviewers, whose constructive comments greatly

374 improved the ms. This work was supported by the Natural Sciences and Engineering Council of 375 Canada (NSERC) Discovery Grants to BJS and MAL.

\section{References}

378

Anagnostou, C., Dorsch, M., Rohlfs, M., 2010. Influence of dietary yeasts on Drosophila melanogaster life-history traits. Entomol. Exp. Appl. 136, 1-11.

Ashburner, M., Golic, K., Hawley, S.H., 2005. Drosophila: A Laboratory Handbook. Cold Spring Harbor Laboratory Press, New York.

Bazinet, A.L., Marshall, K.E., MacMillan, H.A., Williams, C.M., Sinclair, B.J., 2010. Rapid changes in desiccation resistance in Drosophila melanogaster are facilitated by changes in cuticular permeability. J. Insect Physiol. 56, 2006-2012.

Becher, P.G., Flick, G., Rozpędowska, E., Schmidt, A., Hagman, A., Lebreton, S., Larsson, M.C., Hansson, B.S., Piškur, J., Witzgall, P., Bengtsson, M., 2012. Yeast, not fruit volatiles mediate Drosophila melanogaster attraction, oviposition and development. Funct. Ecol. 26, 822-828.

Ben Ami, E., Yuval, B., Jurkevitch, E., 2010. Manipulation of the microbiota of mass-reared Mediterranean fruit flies Ceratitis capitata (Diptera: Tephritidae) improves sterile male sexual performance. ISME J. 4, 28-37.

Benjamini, Y., Hochberg, Y., 1995. Controlling the false discovery rate - a practical and powerful approach to multiple testing. J. R. Stat. Soc. B 57, 289-300.

Bradley, T.J., 2009. Animal Osmoregulation. Oxford University Press, New York.

Broderick, N.A., Buchon, N., Lemaitre, B., 2014. Microbiota-induced changes in Drosophila melanogaster host gene expression and gut morphology. mBio 5, e01117-01114. 
Broderick, N.A., Lemaitre, B., 2012. Gut-associated microbes of Drosophila melanogaster. Gut Microbes 3, 307-321.

Brummel, T., Ching, A., Seroude, L., Simon, A.F., Benzer, S., 2004. Drosophila lifespan enhancement by exogenous bacteria. Proc. Natl. Acad. Sci. U.S.A. 101, 12974-12979.

Chandler, J.A., Eisen, J.A., Kopp, A., 2012. Yeast Communities of Diverse Drosophila Species: Comparison of Two Symbiont Groups in the Same Hosts. Appl. Environ. Microbiol. 78, 7327-7336.

Coon, K.L., Vogel, K.J., Brown, M.R., Strand, M.R., 2014. Mosquitoes rely on their gut microbiota for development. Mol. Ecol. 23, 2727-2739.

Dillon, R.J., Charnley, A.K., 1986. Inhibition of Metarhizium anisopliae by the gut bacterial flora of the desert locust, Schistocerca gregaria - evidence for an antifungal toxin. J. Invertebr. Pathol. 47, 350-360.

Dobzhansky, T., Cooper, D.M., Phaff, H.J., Knapp, E.P., Carson, H.L., 1956. Studies on the ecology of Drosophila in the Yosemite area of California 4. Differential attraction od species of Drosophila to different species of yeasts. Ecology 37, 544-550.

Douglas, A.E., 2015. Multiorganismal Insects: Diversity and Function of Resident Microorganisms. Annu. Rev. Entomol. 60, 17-34.

Douglas, A.E., 2018a. The Drosophila model for microbiome research. Lab Anim. 47, 157-164.

Douglas, A.E., 2018b. Fundamentals of Microbiome Science. Princeton University Press, Princeton.

Engel, P., Moran, N.A., 2013. The gut microbiota of insects - diversity in structure and function. FEMS Microbiol. Rev. 37, 699-735.

Gefen, E., Marlon, A.J., Gibbs, A.G., 2006. Selection for desiccation resistance in adult Drosophila melanogaster affects larval development and metabolite accumulation. J. Exp. Biol. 209, 3293-3300.

Gibbs, A.G., 2002. Water balance in desert Drosophila: lessons from non-charismatic microfauna. Comp. Biochem. Physiol. A 133, 781-789.

Gibbs, A.G., Chippindale, A.K., Rose, M.R., 1997. Physiological mechanisms of evolved desiccation resistance in Drosophila melanogaster. J. Exp. Biol. 200, 1821-1832.

Gibbs, A.G., Fukuzato, F., Matzkin, L.M., 2003. Evolution of water conservation mechanisms in Drosophila. J. Exp. Biol. 206, 1183-1192. 
Gibbs, A.G., Matzkin, L.M., 2001. Evolution of water balance in the genus Drosophila. J. Exp. Biol. 204, 2331-2338.

Hadley, N.F., 1994. Water Relations of Terrestrial Arthropods. Academic Press, San Diego.

Harrison, J.F., Woods, H.A., Roberts, S.P., 2012. Ecological and Environmental Physiology of Insects. Oxford, New York.

Henry, Y., Colinet, H., 2018. Microbiota disruption leads to reduced cold tolerance in Drosophila flies. Sci. Nat. 105, 59.

Hoang, D., Kopp, A., Chandler, J.A., 2015. Interactions between Drosophila and its natural yeast symbionts - Is Saccharomyces cerevisiae a good model for studying the fly-yeast relationship? PeerJ 3, e1116.

Hoffmann, A.A., 1990. Acclimation for desiccation resistance in Drosophila melanogaster and the association between acclimation responses and genetic variation. J. Insect Physiol. 36, 885-891.

Jakobs, R., Gariepy, T.D., Sinclair, B.J., 2015. Adult plasticity of cold tolerance in a continentaltemperate population of Drosophila suzukii. J. Insect Physiol. 79, 1-9.

Jiménez Padilla, Y., 2016. Effects of gut-associated yeasts on Drosophila melanogaster performance. M.Sc. Thesis, University of Western Ontario. https://ir.lib.uwo.ca/etd/4285/.

Judd, A.M., Matthews, M.K., Hughes, R., Veloz, M., Sexton, C.E., Chaston, J.M., 2018. Bacterial methionine metabolism genes influence Drosophila melanogaster starvation resistance. Appl. Env. Microbiol. 84, e00662-00618.

Klepsatel, P., Prochazka, E., Galikova, M., 2018. Crowding of Drosophila larvae affects lifespan and other life-history traits via reduced availability of dietary yeast. Exp. Gerontol. 110, 298-308.

Lachance, M.-A., Gilbert, D.G., Starmer, W.T., 1995. Yeast communities associated with Drosophila species and related flies in an eastern oak-pine forest: A comparison with western communities. J. Industr. Microbiol. 14, 484-494.

Markow, T.A., O'Grady, P., 2005. Drosophila: A guide to species identification and use. Academic Press, London.

Marshall, K.E., Sinclair, B.J., 2010. Repeated stress exposure results in a survival-reproduction trade-off in Drosophila melanogaster. Proc. R. Soc. B 277, 963-969. 
McCluney, K.E., Sabo, J.L., 2010. Tracing Water Sources of Terrestrial Animal Populations with Stable Isotopes: Laboratory Tests with Crickets and Spiders. PLoS One 5, 11.

McFall-Ngai, M., Hadfield, M.G., Bosch, T.C., Carey, H.V., Domazet-Loso, T., Douglas, A.E., Dubilier, N., Eberl, G., Fukami, T., Gilbert, S.F., Hentschel, U., King, N., Kjelleberg, S., Knoll, A.H., Kremer, N., Mazmanian, S.K., Metcalf, J.L., Nealson, K., Pierce, N.E., Rawls, J.F., Reid, A., Ruby, E.G., Rumpho, M., Sanders, J.G., Tautz, D., Wernegreen, J.J., 2013. Animals in a bacterial world, a new imperative for the life sciences. Proc. Natl. Acad. Sci. U.S.A. 110, 3229-3236.

Newell, P.D., Chaston, J.M., Wang, Y., Winans, N.J., Sannino, D.R., Wong, A.C.N., Dobson, A.J., Kagle, J., Douglas, A.E., 2014. In vivo function and comparative genomic analyses of the Drosophila gut microbiota identify candidate symbiosis factors. Front. Microbiol. 5, 576.

Peterson, B.F., Scharf, M.E., 2016. Lower Termite Associations with Microbes: Synergy, Protection, and Interplay. Front. Microbiol. 7, 422.

Phaff, H.J., Miller, M.W., Shifrine, M., 1956. The taxonomy of yeasts isolated from Drosophila in the Yosemite region of California. Antonie van Leeuwenhoek 22, 145-161.

Schretter, C.E., Vielmetter, J., Bartos, I., Marka, Z., Marka, S., Argade, S., Mazmanian, S.K., 2018. A gut microbial factor modulates locomotor behaviour in Drosophila. Nature 563, 402-406.

Schwab, D.B., Riggs, H.E., Newton, I.L.G., Moczek, A.P., 2016. Developmental and Ecological Benefits of the Maternally Transmitted Microbiota in a Dung Beetle. Amer. Nat. 188, 679692.

Scully, E.D., Geib, S.M., Carlson, J.E., Tien, M., McKenna, D., Hoover, K., 2014. Functional genomics and microbiome profiling of the Asian longhorned beetle (Anoplophora glabripennis) reveal insights into the digestive physiology and nutritional ecology of wood feeding beetles. BMC Genomics 15, 1096.

Shin, S.C., Kim, S.-H., You, H., Kim, B., Kim, A.C., Lee, K.-A., Yoon, J.-H., Ryu, J.-H., Lee, W.-J., 2011. Drosophila Microbiome Modulates Host Developmental and Metabolic Homeostasis via Insulin Signaling. Science 334, 670-674.

Starmer, W.T., Fogleman, J.C., 1986. Coadaptation of Drosophila and yeasts in their natural habitat. J. Chem. Ecol. 12, 1037-1055. 
Stinziano, J.R., Sové, R.J., Rundle, H.D., Sinclair, B.J., 2015. Rapid desiccation hardening changes the cuticular hydrocarbon profile of Drosophila melanogaster. Comp. Biochem. Physiol. A 180, 38-42.

Vijendravarma, R.K., Narasimha, S., Kawecki, T.J., 2010. Effects of parental larval diet on egg size and offspring traits in Drosophila. Biol. Lett. 6, 238-241.

Wada-Katsumata, A., Zurek, L., Nalyanya, G., Roelofs, W.L., Zhang, A.J., Schal, C., 2015. Gut bacteria mediate aggregation in the German cockroach. Proc. Natl. Acad. Sci. U.S.A. 112, 15678-15683.

Welte, C.U., de Graaf, R.M., van den Bosch, T.J.M., Op den Camp, H.J.M., van Dam, N.M., Jetten, M.S.M., 2016. Plasmids from the gut microbiome of cabbage root fly larvae encode SaxA that catalyses the conversion of the plant toxin 2-phenylethyl isothiocyanate. Environ. Microbiol. 18, 1379-1390.

Wong, A.C.N., Dobson, A.J., Douglas, A.E., 2014. Gut microbiota dictates the metabolic response of Drosophila to diet. J. Exp. Biol. 217, 1894-1901.

Wong, C.N.A., Ng, P., Douglas, A.E., 2011. Low-diversity bacterial community in the gut of the fruitfly Drosophila melanogaster. Environ. Microbiol. 13, 1889-1900. 
Figure 1 - Survival of adult Drosophila melanogaster under desiccating conditions when axenic

509 or inoculated as adults with three yeast species, (A) Pichia kluyveri, (B) Lachancea kluyveri, and 510 (C) Saccharomyces cerevisiae. Mean ( \pm SD) survival time; Males: dashed lines, square symbols,

511 Females: solid lines, diamond symbols. There were no significant differences between axenic 512 and gnotobiotic flies, and no sex $\times$ treatment interactions; $\uparrow$ indicates significant differences

513 between sexes $(\mathrm{P}<0.05)$. Sample sizes: P. kluyveri - 33 females, 26 males/treatment; L. kluyveri 51462 females, 61 males/treatment; S. cerevisiae 33 females, 32 males/treatment. See Table 1 for 515 results of statistical analyses.

517 Figure 2 - Effect of yeast inoculation as adults on water balance parameters of Drosophila 518 melanogaster under desiccating conditions. Mean \pm SD shown for male (dashed lines, square 519 symbols) and female (solid lines, diamonds) inoculated with Pichia kluyveri (A,B,C), Lachancea 520 kluyveri (D,E,F), or Saccharomyces cerevisiae $(\mathrm{G}, \mathrm{H}, \mathrm{I})$. There were no significant sex $\times$ treatment 521 interactions. * and $\uparrow$ indicate significant differences between gnotobiotic and axenic-treated flies, 522 and between sexes, respectively $(\mathrm{P}<0.05)$. Statistics in Table 1; these parameters are separated by 523 cohort in Fig. S6.

525 Figure 3 - Survival of adult Drosophila melanogaster under desiccating conditions after rearing 526 under axenic conditions or with three yeast species, (A, D) Pichia kluyveri, (B, E) Lachancea 527 kluyveri, and (C, F) Saccharomyces cerevisiae which are alive (A, B, C) or heat-killed (D, E, F). 528 Mean $( \pm \mathrm{SD})$ survival time; Males: dashed lines, square symbols, Females: solid lines, diamond 
529 symbols. There were no significant sex $\times$ treatment interactions; * indicates significant

530 difference between axenic and gnotobiotic flies, $\uparrow$ indicates significant differences between sexes

531 (P<0.05). Live yeasts sample sizes: P. kluyveri - 27 females, 32 males/treatment; L. kluyveri - 31

532 females, 34 males/treatment; S. cerevisiae 31 females, 32 males/treatment. Dead yeast sample

533 sizes: P. kluyveri - 23 females, 26 males/treatment; L. kluyveri - 24 females, 24 males/treatment;

534 S. cerevisiae 25 females, 24 males. See Tables 2 and 3 for statistics.

Figure 4 - Effect of rearing with live yeast on water balance parameters of adult Drosophila

537 melanogaster under desiccating conditions. Mean \pm SD shown for male (dashed lines, square

538 symbols) and female (solid lines, diamonds) inoculated with Pichia kluyveri (A,B,C), Lachancea

539 kluyveri $(\mathrm{D}, \mathrm{E}, \mathrm{F})$, or Saccharomyces cerevisiae $(\mathrm{G}, \mathrm{H}, \mathrm{I})$. Sex $\times$ treatment interactions were

540 significant for initial water content for P. kluyveri and water loss rate for S. cerevisiae. * and $\dagger$

541 indicate significant differences between gnotobiotic and axenic-treated flies, and between sexes,

542 respectively $(\mathrm{P}<0.05)$. Statistics in Table 2.

544 Figure 5 - Effect of rearing with heat-killed yeasts on water balance parameters of adult

545 Drosophila melanogaster under desiccating conditions. Mean \pm SD shown for male (dashed

546 lines, square symbols) and female (solid lines, diamonds) inoculated with Pichia kluyveri

547 (A,B,C), Lachancea kluyveri (D,E,F), or Saccharomyces cerevisiae (G,H,I). There were no

548 significant sex $\times$ treatment interactions.$*$ and $\dagger$ indicate significant differences between

549 gnotobiotic and axenic-treated flies, and between sexes, respectively; Statistics in Table 3. 


\begin{tabular}{|c|c|c|c|}
\hline \multicolumn{4}{|c|}{ Initial Water Content } \\
\hline Effect & Pichia kluyveri & Lachancea kluyveri & Saccharomyces cerevisiae \\
\hline Sex & $F_{1,123}=13.5, P<0.01$ & $F_{1,205}=165.1, P<0.01$ & $F_{1,118}=44.2, P<0.01$ \\
\hline Cohort & $\mathrm{F}_{1,122}=0.3, \mathrm{P}=0.67$ & $\mathrm{~F}_{1,203}=0.1, \mathrm{P}=0.84$ & - \\
\hline Dry Mass & $F_{1,123}=14.0, P<0.01$ & $F_{1,205}=107.8, P<0.01$ & $F_{1,118}=79.8, P<0.01$ \\
\hline Treatment & $\mathrm{F}_{1,121}=0.3, \mathrm{P}=0.43$ & $\mathrm{~F}_{1,204}=1.6, \mathrm{P}=0.30$ & $\mathrm{~F}_{1,118}=3.9, \mathrm{P}=0.10$ \\
\hline Sex $\times$ Treatment & $\mathrm{F}_{1,120}=0.5, \mathrm{P}=0.61$ & $\mathrm{~F}_{1,202}=0.1, \mathrm{P}=0.79$ & $\mathrm{~F}_{1,117}=0.4, \mathrm{P}=0.62$ \\
\hline \multicolumn{4}{|c|}{ Water Content at death } \\
\hline Effect & Pichia kluyveri & Lachancea kluyveri & Saccharomyces cerevisiae \\
\hline Sex & $\mathrm{F}_{1,122}=1.9, \mathrm{P}=0.26$ & $F_{1,204}=13.8, P<0.01$ & $F_{1,120}=27.7, P<0.01$ \\
\hline Cohort & $F_{1,123}=47.7, P<0.01$ & $F_{1,204}=162.1, P<0.01$ & - \\
\hline Dry Mass & $F_{1,123}=29.1, P<0.01$ & $F_{1,204}=13.8, P<0.01$ & $\mathrm{~F}_{1,119}=3.9, \mathrm{P}=0.62$ \\
\hline Treatment & $\mathrm{F}_{1,121}=1.1, \mathrm{P}=0.44$ & $\mathrm{~F}_{1,203}=3.3, \mathrm{P}=0.12$ & $\mathrm{~F}_{1,118}=0.9, \mathrm{P}=0.48$ \\
\hline Sex $\times$ Treatment & $\mathrm{F}_{1,120}=2.3, \mathrm{P}=0.20$ & $\mathrm{~F}_{1,202}=0.9, \mathrm{P}=0.46$ & $\mathrm{~F}_{1,117}=3.8, \mathrm{P}=0.11$ \\
\hline \multicolumn{4}{|l|}{ Water Loss Rate } \\
\hline Effect & Pichia kluyveri & Lachancea kluyveri & Saccharomyces cerevisiae \\
\hline Sex & $\mathrm{F}_{1,124}=1.7, \mathrm{P}=0.30$ & $F_{1,204}=11.4, P<0.01$ & $\mathrm{~F}_{1,118}=0.04, \mathrm{P}=0.88$ \\
\hline Cohort & $\mathrm{F}_{1,122}=0.3, \mathrm{P}=0.70$ & $F_{1,204}=144.4, P<0.01$ & - \\
\hline Dry Mass & $\mathrm{F}_{1,121}=0.3, \mathrm{P}=0.67$ & $\mathrm{~F}_{1,204}=4.8, \mathrm{P}=0.06$ & $F_{1,119}=6.4 P=0.02$ \\
\hline Treatment & $\mathrm{F}_{1,123}=0.4, \mathrm{P}=0.62$ & $\mathrm{~F}_{1,203}=3.0, \mathrm{P}=0.14$ & $\mathrm{~F}_{1,119}=4.3, \mathrm{P}=0.08$ \\
\hline Sex $\times$ Treatment & $\mathrm{F}_{1,120}=1.3, \mathrm{P}=0.37$ & $\mathrm{~F}_{1,202}=0.6, \mathrm{P}=0.58$ & $\mathrm{~F}_{1,117}=0.0001, \mathrm{P}=0.99$ \\
\hline \multicolumn{4}{|c|}{ Survival time under desiccating conditions } \\
\hline Effect & Pichia kluyveri & Lachancea kluyveri & Saccharomyces cerevisiae \\
\hline Sex & $F_{1,122}=15.9, P<0.01$ & $F_{1,204}=31.8, P<0.01$ & $F_{1,119}=26.6, P<0.001$ \\
\hline Cohort & $F_{1,122}=8.4, P=0.01$ & $F_{1,204}=50.7, P<0.01$ & - \\
\hline Dry Mass & $\mathrm{F}_{1,122}=4.1, \mathrm{P}=0.10$ & $F_{1,204}=14.8, P<0.01$ & $F_{1,119}=22.4, P<0.01$ \\
\hline Treatment & $\mathrm{F}_{1,121}=2.4, \mathrm{P}=0.20$ & $\mathrm{~F}_{1,203}=0.3, \mathrm{P}=0.68$ & $\mathrm{~F}_{1,118}=0.02, \mathrm{P}=0.93$ \\
\hline Sex $\times$ Treatment & $\mathrm{F}_{1,120}=2.3, \mathrm{P}=0.20$ & $\mathrm{~F}_{1,202}=0.0001, \mathrm{P}=0.99$ & $\mathrm{~F}_{1,117}=0.6, \mathrm{P}=0.56$ \\
\hline
\end{tabular}

Table 1 - Summary of Analysis of Covariance of water balance and desiccation survival of axenic and gnotobiotic D. melanogaster inoculated with Pichia kluyveri, Lachancea kluyveri, and Saccharomyces cerevisiae. Cohorts are separated according to date of the experiment (all S. cerevisiae experiments were conducted on the same day, so there is no cohort term in the model); significant terms are in bold typeface; all p-values have been subjected to a study-wide Benjamini-Hochberg FDR correction. 


\begin{tabular}{|c|c|c|c|}
\hline \multicolumn{4}{|c|}{ Initial Water Content } \\
\hline Effect & Pichia kluyveri & Lachancea kluyveri & Saccharomyces cerevisiae \\
\hline Sex & $F_{1,115}=116.4, P<0.01$ & $F_{1,120}=89.9, P<0.01$ & $F_{1,120}=32.5, P<0.01$ \\
\hline Dry Mass & $\mathrm{F}_{1,114}=1.6, \mathrm{P}=0.33$ & $F_{1,120}=13.8, P<0.01$ & $\mathrm{~F}_{1,119}=0.4, \mathrm{P}=0.62$ \\
\hline Treatment & $F_{1,115}=48.3, P<0.01$ & $F_{1,120}=154.5, P<0.01$ & $F_{1,120}=61.0, P<0.01$ \\
\hline Sex $\times$ Treatment & $F_{1,115}=6.9, P=0.02$ & $\mathrm{~F}_{1,120}=7.9, \mathrm{P}=0.11$ & $\mathrm{~F}_{1,118}=0.1, \mathrm{P}=0.80$ \\
\hline \multicolumn{4}{|c|}{ Water Content at death } \\
\hline Effect & Pichia kluyveri & Lachancea kluyveri & Saccharomyces cerevisiae \\
\hline Sex & $F_{1,115}=46.7, P<0.01$ & $F_{1,121}=12.8, P<0.01$ & $F_{1,121}=25.6, P<0.01$ \\
\hline Dry Mass & $\mathrm{F}_{1,114}=0.1, \mathrm{P}=0.80$ & $F_{1,121}=7.4, P=0.02$ & $\mathrm{~F}_{1,120}=2.9, \mathrm{P}=0.16$ \\
\hline Treatment & $F_{1,115}=15.5, P<0.01$ & $F_{1,121}=35.4, P<0.01$ & $\mathrm{~F}_{1,119}=1.4, \mathrm{P}=0.35$ \\
\hline Sex $\times$ Treatment & $\mathrm{F}_{1,115}=5.0, \mathrm{P}=0.06$ & $\mathrm{~F}_{1,120}=0.003, \mathrm{P}=0.97$ & $\mathrm{~F}_{1,118}=0.7, \mathrm{P}=0.55$ \\
\hline \multicolumn{4}{|l|}{ Water Loss Rate } \\
\hline Effect & Pichia kluyveri & Lachancea kluyveri & Saccharomyces cerevisiae \\
\hline Sex & $\mathrm{F}_{1,115}=0.4, \mathrm{P}=0.62$ & $\mathrm{~F}_{1,121}=2.5, \mathrm{P}=0.20$ & $F_{1,118}=9.5, P<0.01$ \\
\hline Dry Mass & $\mathrm{F}_{1,116}=0.5, \mathrm{P}=0.60$ & $\mathrm{~F}_{1,122}=0.4, \mathrm{P}=0.65$ & $\mathrm{~F}_{1,118}=4.0, \mathrm{P}=0.10$ \\
\hline Treatment & $\mathrm{F}_{1,117}=3.4, \mathrm{P}=0.12$ & $F_{1,123}=75.1, P<0.01$ & $F_{1,118}=68.8, P<0.01$ \\
\hline Sex $\times$ Treatment & $\mathrm{F}_{1,114}=1.4, \mathrm{P}=0.36$ & $\mathrm{~F}_{1,120}=2.3, \mathrm{P}=0.20$ & $F_{1,118}=11.6, P<0.01$ \\
\hline \multicolumn{4}{|c|}{ Survival time under desiccating conditions } \\
\hline Effect & Pichia kluyveri & Lachancea kluyveri & Saccharomyces cerevisiae \\
\hline Sex & $F_{1,117}=41.0, P<0.01$ & $F_{1,122}=54.1, P<0.01$ & $F_{1,120}=54.9, P<0.01$ \\
\hline Dry Mass & $\mathrm{F}_{1,115}=0.2, \mathrm{P}=0.73$ & $\mathrm{~F}_{1,121}=0.007, \mathrm{P}=0.97$ & $\mathrm{~F}_{1,119}=0.1, \mathrm{P}=0.82$ \\
\hline Treatment & $\mathrm{F}_{1,116}=0.2, \mathrm{P}=0.78$ & $F_{1,122}=64.8, P<0.01$ & $F_{1,120}=37.3, P<0.01$ \\
\hline Sex $\times$ Treatment & $\mathrm{F}_{1,114}=0.8, \mathrm{P}=0.49$ & $\mathrm{~F}_{1,120}=0.7, \mathrm{P}=0.56$ & $\mathrm{~F}_{1,118}=0.9, \mathrm{P}=0.49$ \\
\hline
\end{tabular}

Table 2- Summary of Analysis of Covariance of water balance and desiccation survival of axenic and gnotobiotic D. melanogaster reared with Pichia kluyveri, Lachancea kluyveri, and Saccharomyces cerevisiae. Significant terms are in bold typeface; all p-values have been subjected to a study-wide Benjamini-Hochberg FDR correction. 


\begin{tabular}{|c|c|c|c|}
\hline \multicolumn{4}{|c|}{ Initial Water Content } \\
\hline Effects & Pichia kluyveri & Lachancea kluyveri & Saccharomyces cerevisiae \\
\hline Sex & $F_{1,92}=38.6 P<0.01$ & $F_{1,92}=41.8, P<0.01$ & $F_{1,93}=207.7, P<0.01$ \\
\hline Dry Mass & $F_{1,92}=230.5, P<0.01$ & $F_{1,92}=69.7, P<0.01$ & $F_{1,92}=53.8, P<0.01$ \\
\hline Treatment & $\mathrm{F}_{1,91}=0.13, \mathrm{P}=0.12$ & $\mathrm{~F}_{1,91}=3.5, \mathrm{P}=0.11$ & $F_{1,92}=9.1, P<0.01$ \\
\hline Sex $\times$ Treatment & $\mathrm{F}_{1,91}=0.005, \mathrm{P}=0.97$ & $\mathrm{~F}_{1,90}=0.003, \mathrm{P}=0.97$ & $\mathrm{~F}_{1,91}=2.8, \mathrm{P}=0.17$ \\
\hline \multicolumn{4}{|c|}{ Water Content at death } \\
\hline Effects & Pichia kluyveri & Lachancea kluyveri & Saccharomyces cerevisiae \\
\hline Sex & $\mathrm{F}_{1,92}=4.9, \mathrm{P}=0.06$ & $F_{1,93}=5.6, P=0.04$ & $\mathrm{~F}_{1,92}=2.3, \mathrm{P}=0.20$ \\
\hline Dry Mass & $F_{1,92}=10.4, P<0.01$ & $\mathrm{~F}_{1,92}=2.7, \mathrm{P}=0.19$ & $F_{1,93}=23.7, P<0.01$ \\
\hline Treatment & $F_{1,92}=26.4, P<0.01$ & $\mathrm{~F}_{1,91}=1.5, \mathrm{P}=0.33$ & $F_{1,93}=39.2, P<0.01$ \\
\hline Sex $\times$ Treatment & $\mathrm{F}_{1,91}=2.1, \mathrm{P}=0.23$ & $\mathrm{~F}_{1,90}=3.3, \mathrm{P}=0.12$ & $\mathrm{~F}_{1,91}=0.5, \mathrm{P}=0.62$ \\
\hline \multicolumn{4}{|l|}{ Water Loss Rate } \\
\hline Effects & Pichia kluyveri & Lachancea kluyveri & Saccharomyces cerevisiae \\
\hline Sex & $F_{1,92}=11.1, P<0.01$ & $F_{1,93}=6.8, P=0.02$ & $F_{1,93}=16.8, P<0.01$ \\
\hline Dry Mass & $\mathrm{F}_{1,92}=9.4, \mathrm{P}<0.01$ & $\mathrm{~F}_{1,92}=0.2, \mathrm{P}=0.73$ & $\mathrm{~F}_{1,92}=3.4, \mathrm{P}=0.12$ \\
\hline Treatment & $F_{1,92}=15.2, P<0.01$ & $\mathrm{~F}_{1,91}=0.1, \mathrm{P}=0.86$ & $F_{1,93}=16.8, P=0.04$ \\
\hline Sex $\times$ Treatment & $\mathrm{F}_{1,91}=0.6, \mathrm{P}=0.57$ & $\mathrm{~F}_{1,90}=0.004, \mathrm{P}=0.97$ & $\mathrm{~F}_{1,91}=0.7, \mathrm{P}=0.54$ \\
\hline \multicolumn{4}{|c|}{ Survival time under desiccating conditions } \\
\hline Effects & Pichia kluyveri & Lachancea kluyveri & Saccharomyces cerevisiae \\
\hline Sex & $F_{1,93}=71.4, P<0.01$ & $F_{1,92}=54.1, P<0.01$ & $F_{1,92}=5.4, P=0.04$ \\
\hline Dry Mass & $F_{1,93}=36.0, P<0.01$ & $\mathrm{~F}_{1,92}=23.8, \mathrm{P}<0.01$ & $F_{1,92}=104.6, P<0.01$ \\
\hline Treatment & $\mathrm{F}_{1,92}=3.4, \mathrm{P}=0.12$ & $\mathrm{~F}_{1,91}=2.3, \mathrm{P}=0.20$ & $F_{1,92}=5.8, P=0.04$ \\
\hline Sex $\times$ Treatment & $\mathrm{F}_{1,91}=0.1, \mathrm{P}=0.79$ & $\mathrm{~F}_{1,90}=0.5, \mathrm{P}=0.62$ & $\mathrm{~F}_{1,91}=0.3, \mathrm{P}=0.67$ \\
\hline
\end{tabular}

Table 3 - Summary of Analysis of Covariance (ANCOVA) of water balance and desiccation survival of axenic and gnotobiotic $D$. melanogaster reared with heat-killed Pichia kluyveri, Lachancea kluyveri, and Saccharomyces cerevisiae. Significant terms are in bold typeface; all p-values have been subjected to a study-wide Benjamini-Hochberg FDR correction. 

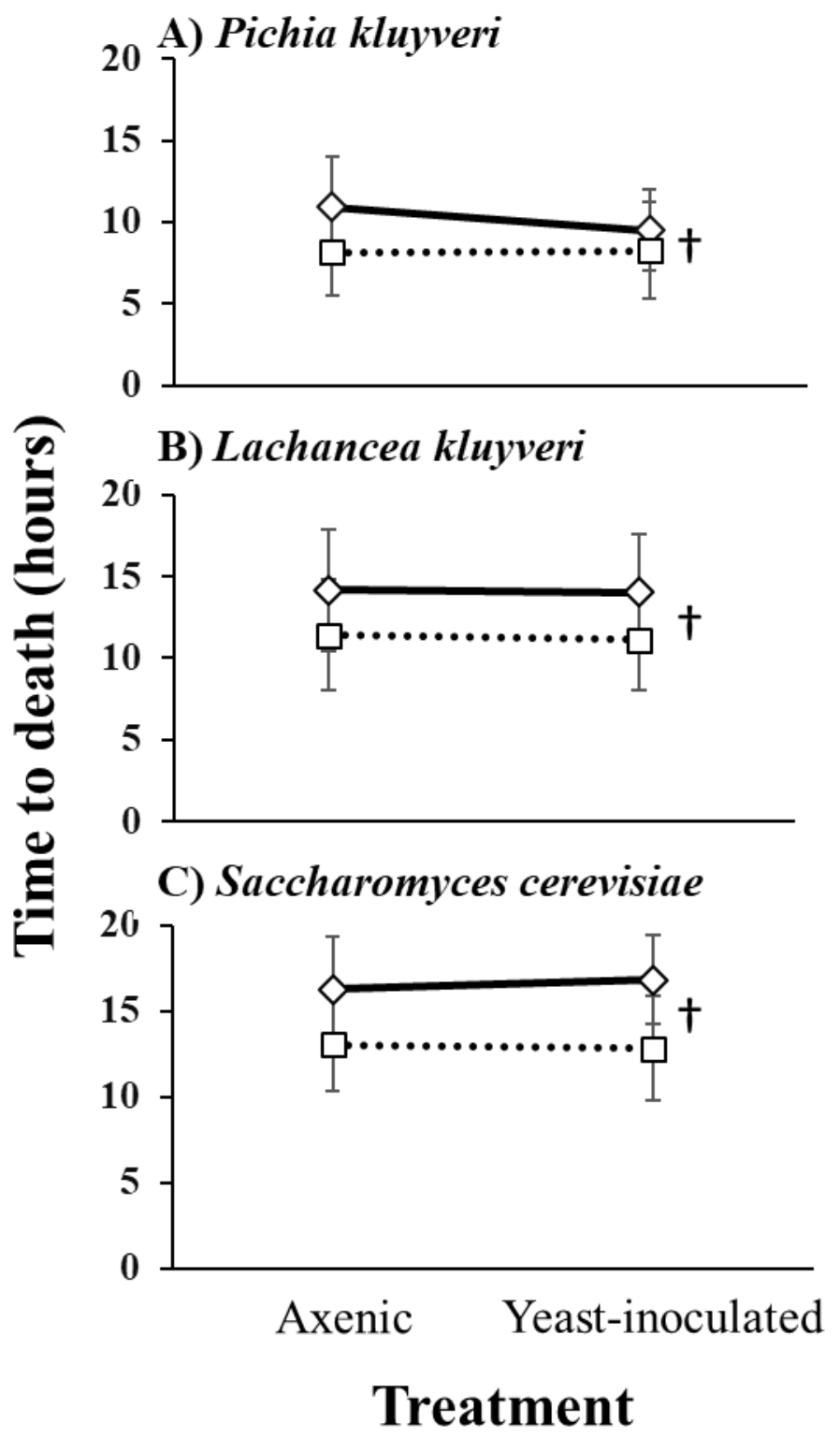

569

570 

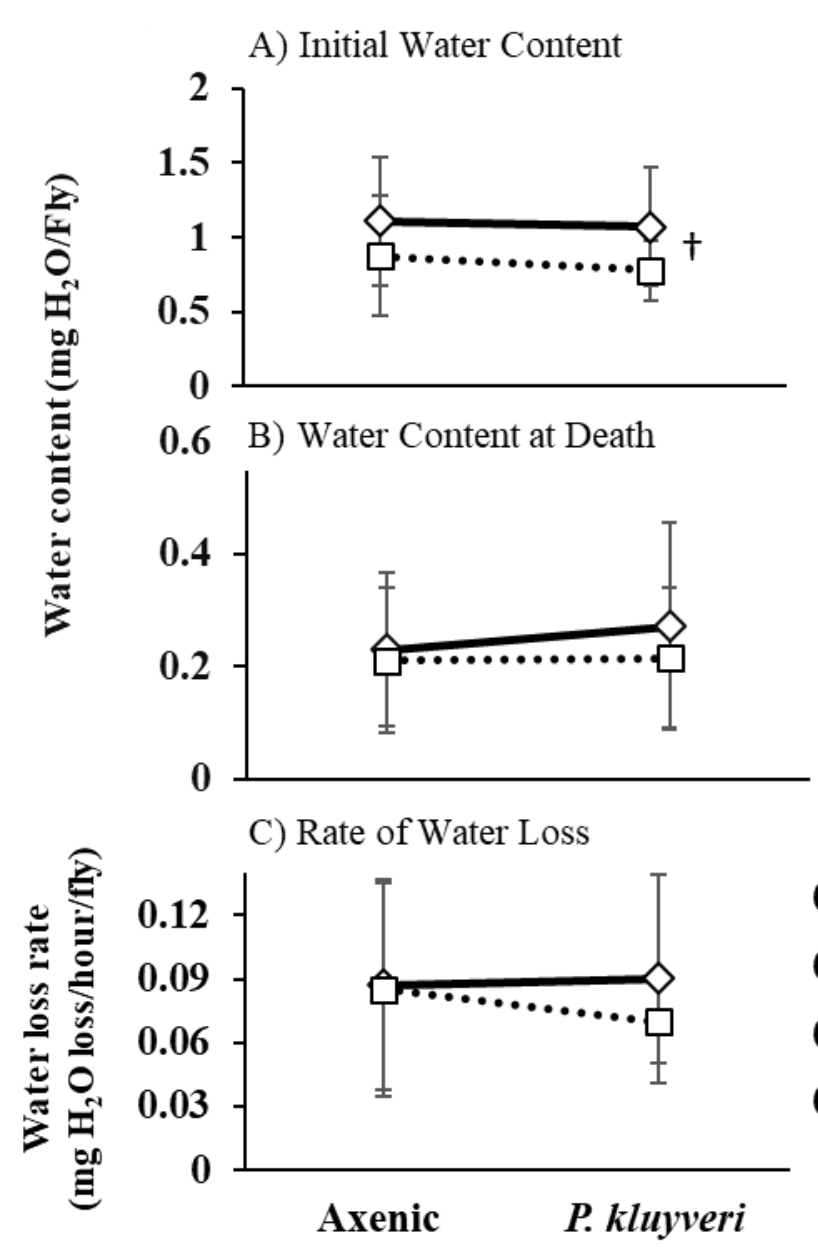

D) Initial Water Content

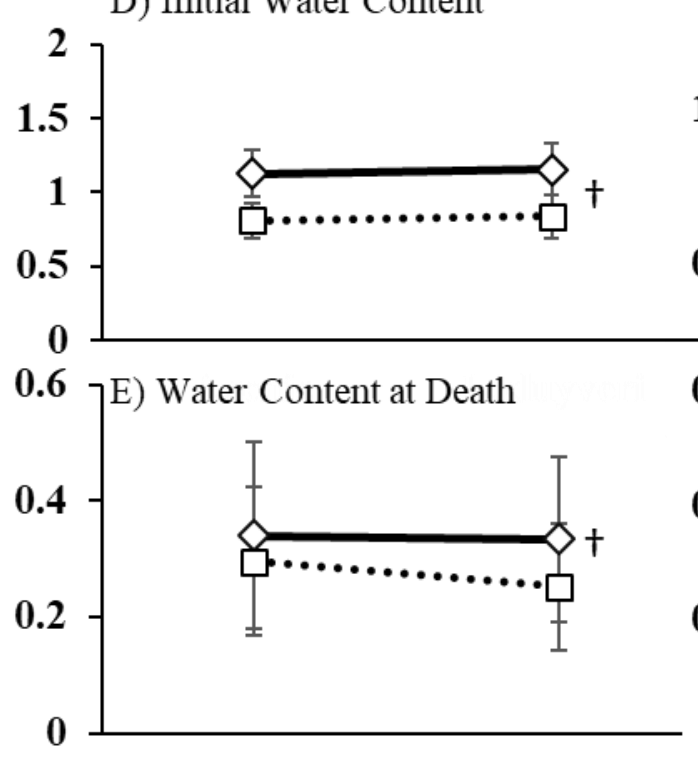

F) Rate of Water Loss

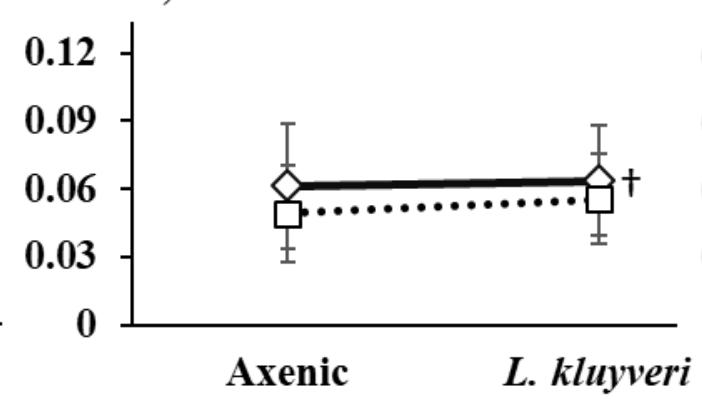

G) Initial Water Content

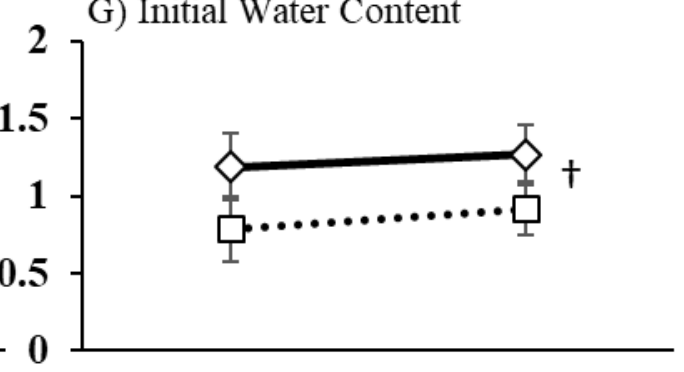

$0.6{ }^{\mathrm{H})}$ Water Content at Death

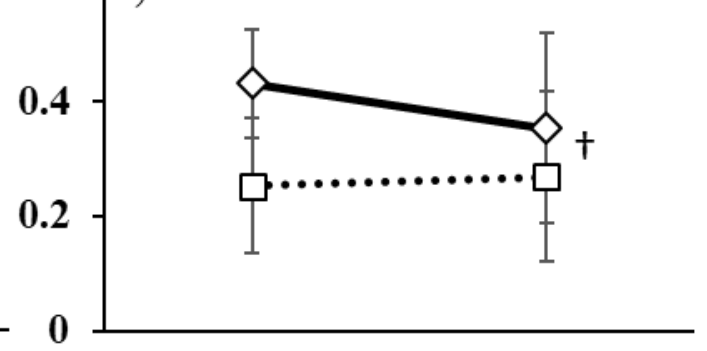

I) Rate of Water Loss 
574 Figure 3

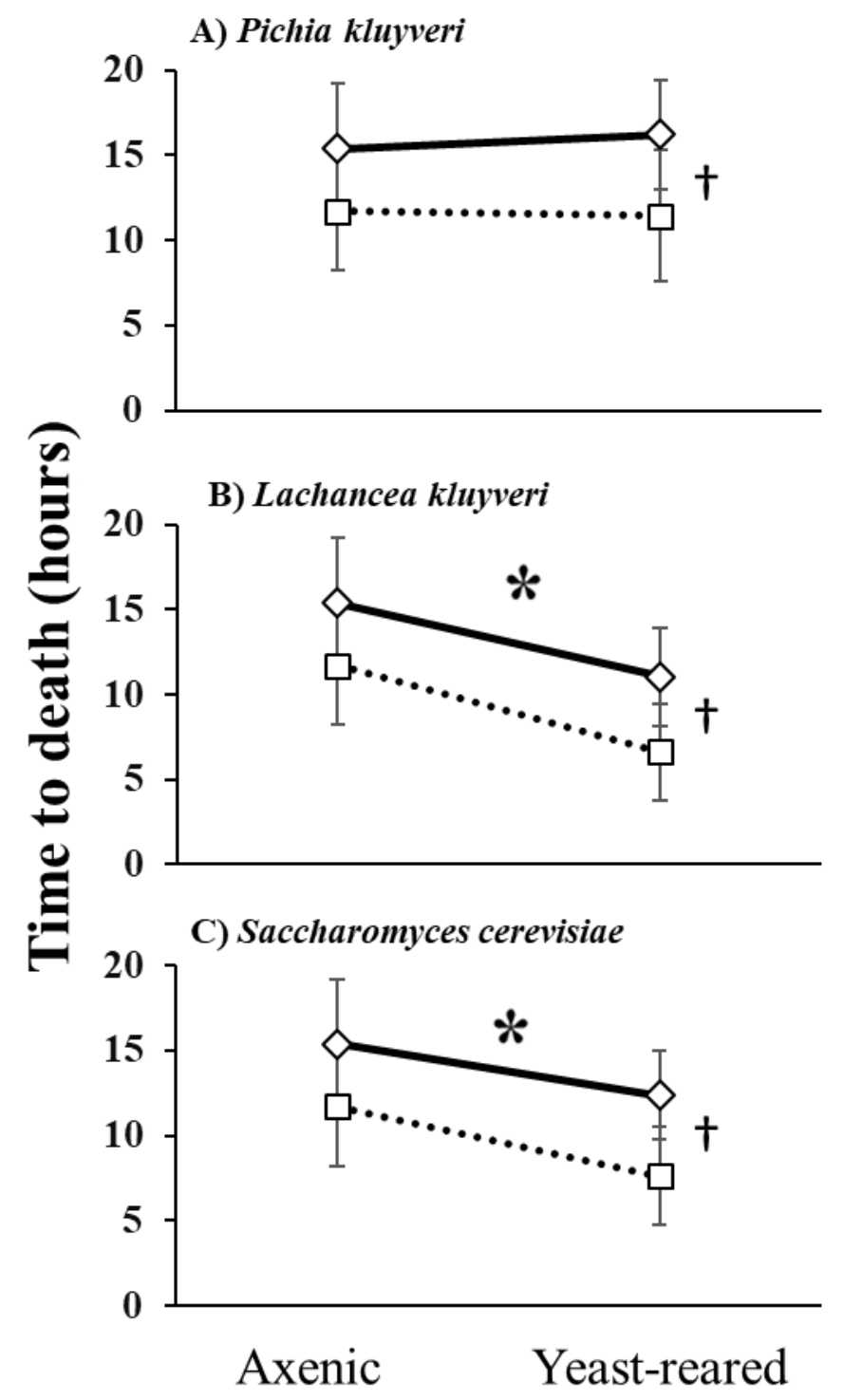

D) Heat-killed Pichia kluyveri
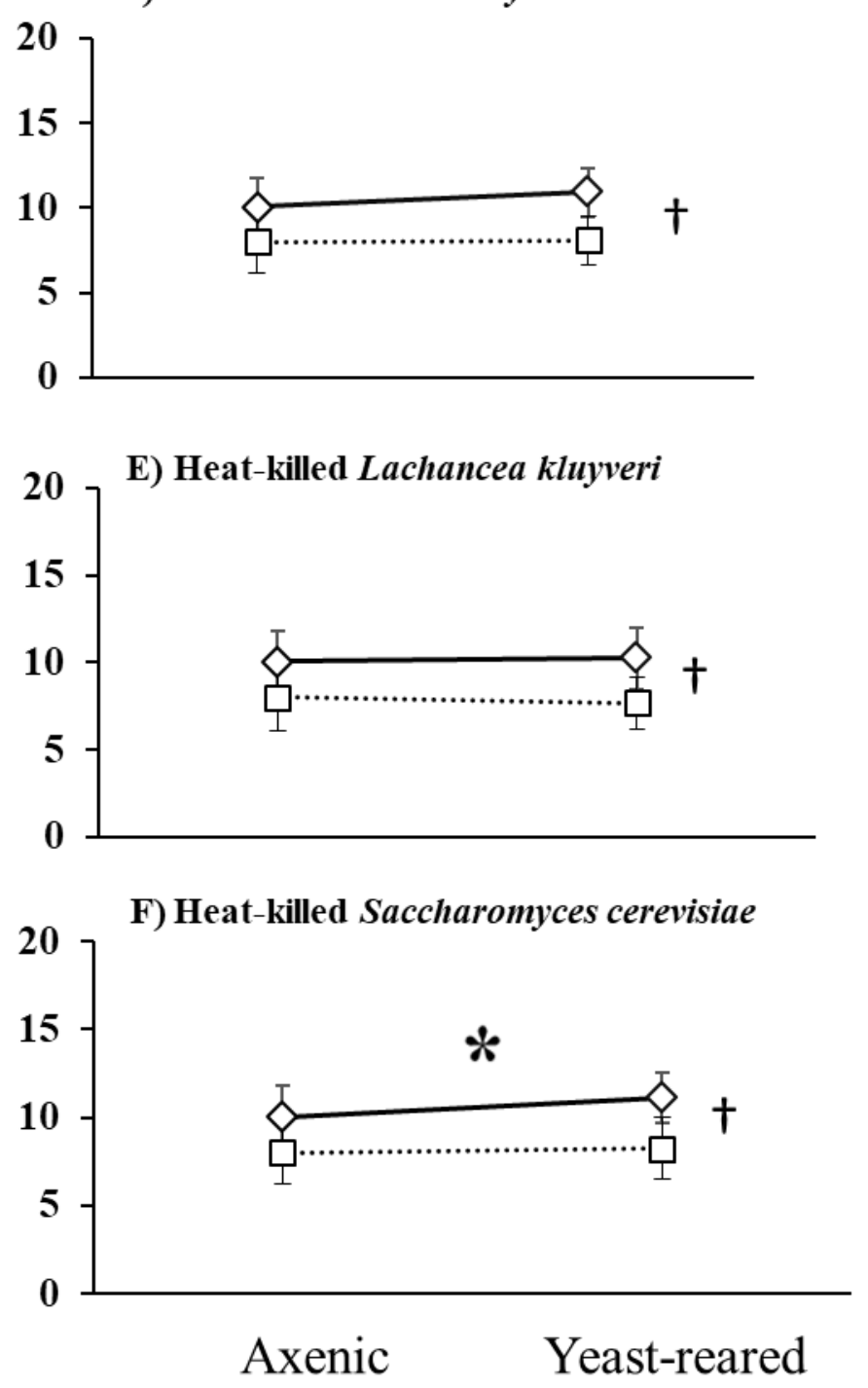

\section{Treatment}

575

576 

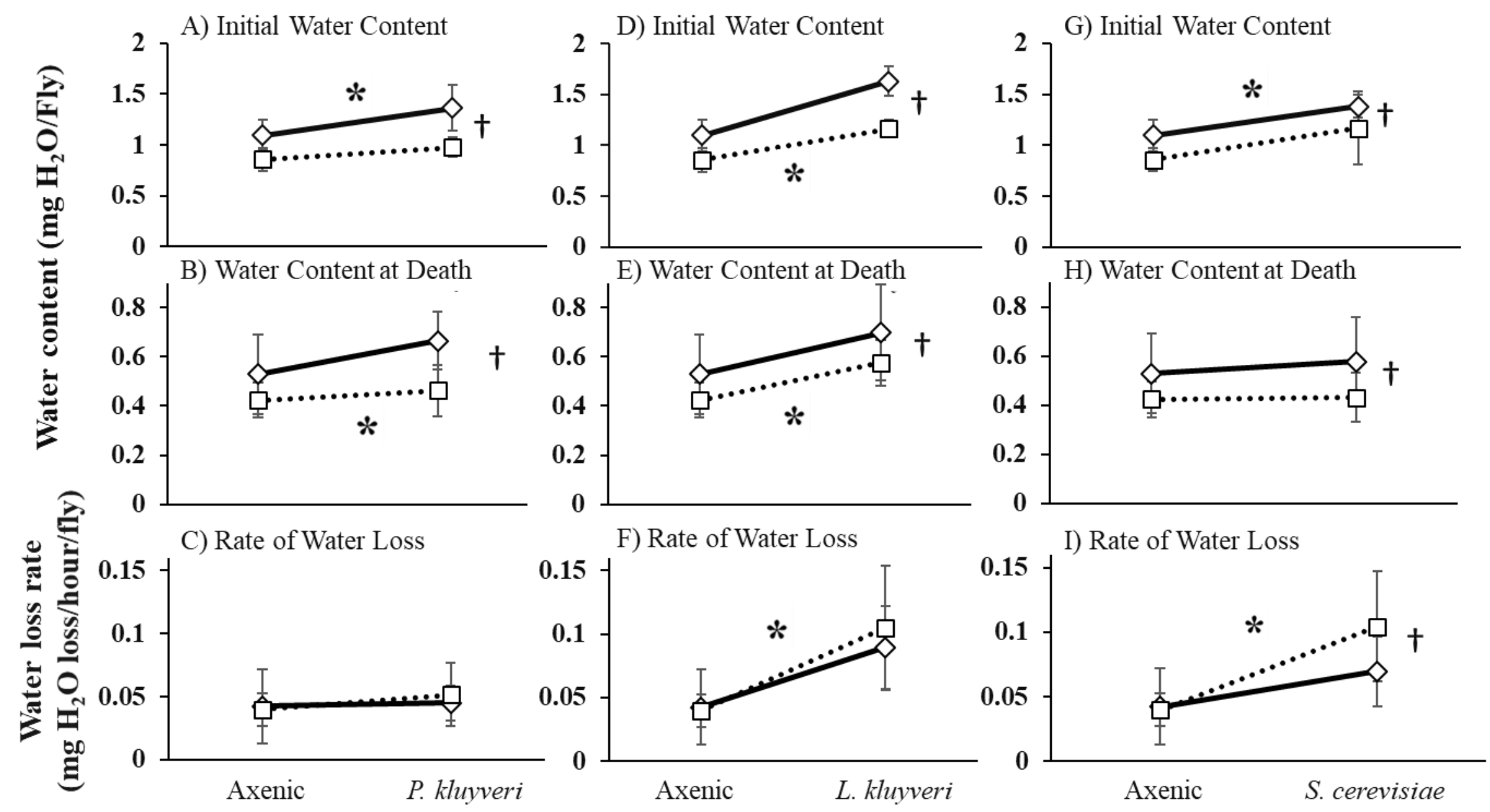

\section{Treatment}



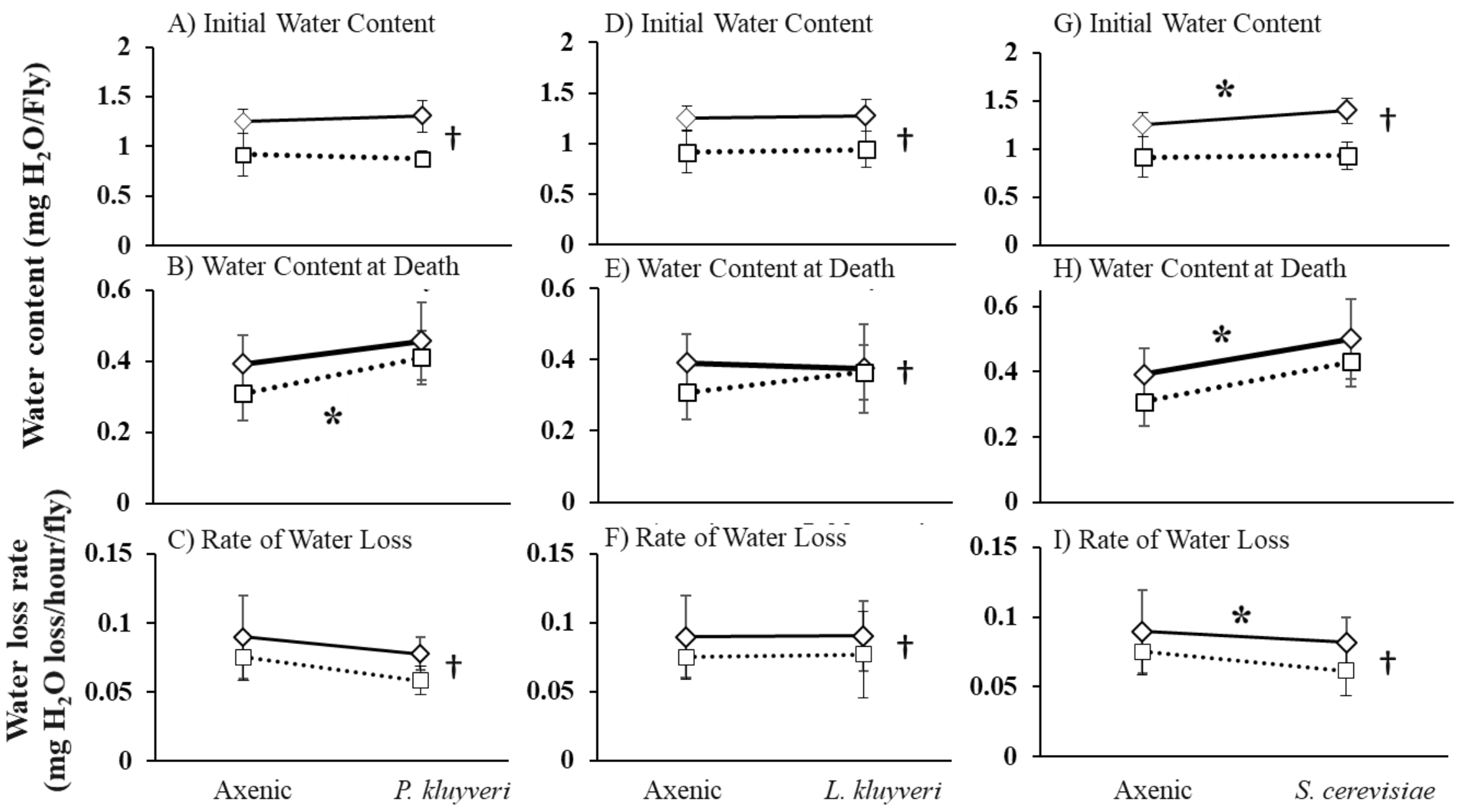

Treatment 
